\title{
A Case of Repeating Transient Increase in Intraocular Pressure by Instability of an Intraocular Lens Implanted in the Capsular Bag
}

\author{
Yukihisa Takada Takayoshi Sumioka Nobuyuki Ishikawa \\ Shingo Yasuda Ryoko Komori Shizuya Saika \\ Department of Ophthalmology, Wakayama Medical University, Wakayama, Japan
}

\section{Keywords}

In-the-bag intraocular lens · Dislocation of an intralocular lens · Repeating increase in intraocular pressure and normalization $\cdot$ In situ scleral refixation

\begin{abstract}
We observed repeated episodes of rapid increases in intraocular pressure (IOP) considered to be caused by an in-the-bag intraocular lens $(\mathrm{IOL})$ instability in a patient with an implanted IOL. As acute glaucoma attack-like increase in IOP was noted in the left eye on November 8, she was admitted to Wakayama Medical University Hospital. The findings at the first examination included an IOP of $62 \mathrm{~mm} \mathrm{Hg}$, instability of a PMMA one-piece IOL, shallow anterior chamber, narrow angle, moderate mydriasis, and loss of pupillary light reaction in the left avitreous eye. On November 15, a 6-mm Hg increase in IOP was observed during 60-min dark room prone provocative testing. After the first examination, the patient perceived pain and reduced visual acuity of the left eye and emergently consulted our hospital twice. Despite miosis, normalization of the anterior chamber depth and IOP with widening of the angle were achieved by resting in the supine position. These episodes were thought to be caused by instability and anterior shift of the IOL. On January 17, 2018, suture fixation of the in-the-bag IOL was performed. The $\mathrm{IOL}$ was fixed by transscleral suturing of the bilateral supporting parts to the sclera. Recurrence of sudden ophthalmalgia, instability of the in-the-bag IOL, and an increase in IOP have not been observed for 1 year after surgical treatment. Instability of an in-the-bag IOL caused
\end{abstract}




\section{Case Reports in Ophthalmology}

repeated acute angle-closure glaucoma-like attacks. The situation was well treated by suturing and fixing the haptics of IOL to the sclera.

\section{Introduction}

Dislocation of an intraocular lens (IOL) implanted during cataract surgery is one of the problems encountered during surgery [1]. Although this complication occurs frequently in eyes with an out-of-the-bag IOL with a partially damaged lens capsule, weakness or rupture of Zinn's zonule could cause displacement of the capsular bag that contains an IOL [2, 3]. Ocular hypertension might be caused by pupillary block due to adhesion of the IOL to the iris [4], dislocation of an out-of-the-bag IOL [5], and vitreous body prolapse after YAG laser posterior capsulotomy for secondary cataract in patients with an in-the-bag IOL [6].

On the other hand, spontaneous dislocation of an in-the-bag IOL was also reported [4]. Its background includes preexisting pseudoexfoliation syndrome, history of uveitis or vitreous surgery or blunt trauma, etc. However, it does not seem to be common that dislocation of an in-the-bag IOL causes an increment of intraocular pressure (IOP) such as acute angle-closure glaucoma, dissimilar to dislocation of a crystalline lens.

In this report, we present a patient with a history of in-the-bag IOL implantation and vitreous surgery who had repeated episodes of a rapid increase of IOP with closure of the anterior chamber angle. The situation was relieved by in situ scleral refixation of the supporting part of the IOL.

\section{Case Presentation}

\section{Patient}

On November 8, 2017, a 63-year-old female presented a shallow anterior chamber and narrow angle, moderate mydriasis, ocular hypertension, ophthalmalgia, and reduced visual acuity in her left eye and was referred to the Department of Ophthalmology, Wakayama Medical University Hospital, Japan. Examinations at the first consultation showed ciliary hyperemia, corneal epithelial edema, moderate mydriasis, loss of light response, IOL instability, closing of the angle (Scheie grade IV), and widening of the depression of the optic disc (vertical C/D ratio: 0.7 ) in the affected eye. Visual acuity was 0.03 (uncorrected), and IOP was $62 \mathrm{~mm}$ Hg (Fig. 1a-c). Secondary cataract was also observed. Vitreous body was absent. In the right eye, no abnormality was noted from the anterior eye to the fundus other than the cataract. Visual acuity was $0.3(0.9 \times \mathrm{S}-1.00 \mathrm{D})$, and IOP was $15 \mathrm{~mm} \mathrm{Hg}$.

The patient's medical history was as follows. She underwent cataract surgery (in-the-bag fixation of PMMA 1-piece IOL) and vitreous surgery of the left eye for cataract and macular edema associated with branch retinal vein occlusion in 2005. She experienced repeated episodes of a sudden increase of IOP (approx. $30 \mathrm{~mm} \mathrm{Hg}$ ).

\section{Clinical Findings}

At the first consultation she received mannitol drop infusion and ocular instillation of pilocarpine hydrochloride in the supine position, which alleviated the corneal edema, miosis, and widening of the angle (Scheie grade III), and normalization of the IOP (20 mm Hg) (Fig. $1 \mathrm{~d}-\mathrm{f}$ ) in the affected eye. On November 15 , the dark room prone provocative testing (60 $\mathrm{min}$ ) resulted in an increment of the IOP by $3 \mathrm{~mm} \mathrm{Hg}$ in the right eye and $6 \mathrm{~mm} \mathrm{Hg}$ in the left eye. 


\section{Case Reports in Ophthalmology}

Thereafter, she developed ophthalmalgia accompanied by shallowing of the anterior chamber and increased IOP on December 9 and December 18. The IOP could be reduced, and ophthalmalgia was resolved by mannitol drip infusion and ocular instillation of pilocarpine hydrochloride during rest in the supine position in association with normalization of the IOP. The patient stated that she had prolonged desk work as a common condition before the appearance of ophthalmalgia, and that it was commonly alleviated or resolved by resting in the supine position and eye massage. On December 18, the anterior chamber in the marginal region was shallow only in the upper direction at the time of emergency consultation (Fig. 1g), and the IOP was reduced by mannitol drip infusion and massage of the region.

The mechanism of the rapid increase in IOP was suggested to be a temporary closure of the angle and pupillary block due to anterior displacement/shift of the IOL associated with inthe-bag IOL instability. Surgery was performed on January 18, 2018, to resolve the IOL instability. Preoperative findings were as follows: the visual acuity of the left eye was $0.3(0.7 \times \mathrm{S}-$ $1.25 \mathrm{D}=\mathrm{C}-0.75 \mathrm{Dax} 110$ ). IOP was $14 \mathrm{~mm} \mathrm{Hg}$ (with the use of pilocarpine hydrochloride) in the affected eye. On static visual field testing (Humphrey 30-2), the MD value was $-9.68 \mathrm{~dB}$, and the corneal epithelial cell density was $2,602 / \mathrm{mm}^{2}$. The axial length was $23.29 \mathrm{~mm}$.

\section{Surgical Procedure}

A conjunctival incision was made in the 1 o'clock direction, a scleral flap was made, and a $27 \mathrm{G}$ trocar for vitreous surgery was placed in the corneal limbus in the $7 \mathrm{o}$ 'clock direction (Fig. 2a) after the irrigation port had been placed in the flat part of the ciliary body in the 5 o'clock direction (Fig. 3a). A 10-0 nylon thread straight needle was advanced under the scleral flap in the 1-o'clock direction (4 mm from the corneal limbus) (Fig. 2b, 3b), led out of the eye through a trocar placed in the cornea on the opposite side (Fig. 2b), and inserted again through the trocar. Then, the thread was applied to the complex of the haptic of the IOL and the capsule. The $27 \mathrm{G}$ needle was advanced under the scleral flap in the 1-o'clock direction, and the straight needle was captured inside the $27 \mathrm{G}$ needle (Fig. 3c) and guided out of the eye (Fig. 2c). The straight needle pushed out of the eye was sutured to the sclera under the scleral flap (Fig. 2d, $3 d$ ). By the same procedure, a conjunctival incision was made in the 7-o'clock direction, a scleral flap was made, a trocar was placed in the corneal limbus of the 1-o'clock direction (Fig. 3d), and the 10-0 nylon thread straight needle was applied via the transscleral method and sutured to the sclera under the scleral flap in the 7-o'clock direction (Fig. 3e). The surgery was ended by removing the irrigation port and suturing the conjunctiva with an 8-0 VICRYL suture (Fig. 3e, f).

\section{Discussion and Conclusion}

Several reports are available about secondary glaucoma in IOL-implanted eyes [5]. Pupillary block by IOL is known to be a cause of a sudden increase in IOP [4]. It was also reported that an anterior shift of the capsular tension ring-IOL complex caused closure of the anterior chamber angle and an increase of IOP [7]. However, there is no report of a patient with repeated episodes of an increase in IOP after in-the-bag IOL implantation with no complications in cataract surgery, as was the case in our patient.

As our patient exhibited IOL instability and an increase in IOP of $6 \mathrm{~mm} \mathrm{Hg}$ during dark room prone provocative testing [8], temporary closure of the angle or pupillary block might have been induced by anterior shift of the IOL in the prone position. As the anterior chamber was shallow only around the upper part of the iris with increased IOP at the emergency 
consultation (December 18, 2017) (Fig. 1g), the temporary increase in IOP was considered to have been caused primarily by compression of the angle by the unstable IOL. The patient reported that ophthalmalgia and ocular hypertension were induced by prolonged desk work in the prone position and relieved by resting in the supine position; therefore, these episodes were considered to support the above mechanism.

We considered resolution of IOL instability or creation of a bypass between the anterior and posterior chambers to be necessary to avoid increases in IOP. Specifically, methods to resolve IOL instability include one-stage or two-stage IOL implantation (intrascleral fixation [9], suturing to the ciliary sulcus [10], etc.) after removing the IOL, and suturing of the supporting parts of the IOL and lenticular capsule to the sclera to strengthen fixation of the unstable IOL. It is necessary to make a wound with a sufficient size for removal of the IOL in procedures that involve its removal, which may increase the risk of surgically induced astigmatism [11]. Moreover, future possible decline in visual function due to IOL decentration or its displacement into the vitreous cavity is of concern. In the method for strengthening the supporting part of the unstable IOL or lenticular capsule by applying a nylon thread, there was the risk of rupture of Zinn's zonule by puncturing the lenticular capsule with a straight needle, but this procedure, which can be performed with a small wound, was selected for our patient because of the lower risk of surgically induced astigmatism and postoperative infection. Our surgical procedure presented here was similar to that reported by Fabrizio et al. [12]. During the postoperative course, the mean corneal astigmatism was $-1.25 \mathrm{D}\left(\mathrm{Ax}^{\circ} 5^{\circ}\right)$ at the first examination (after normalization of ocular pressure by mannitol drip infusion) and $-0.75 \mathrm{D}$ $\left(A x 81^{\circ}\right)$ at the last examination, indicating that surgery-induced astigmatism was escaped. The operation completely resolved the symptom of ophthalmalgia and IOP elevation during prolonged desk work in the prone position with the resolution of IOL instability by the present surgery (Fig. 1h, i).

In summary, we experienced a case of repeated episodes of IOP elevation with angle closure caused by anteriorly displaced in-the-bag IOL. The condition was well managed by in situ scleral refixation of the haptics of the in-the-bag IOL.

\section{Statement of Ethics}

Oral consent to publish this case has been obtained, but written consent has not been obtained. This report does not contain any personal identifying information.

\section{Disclosure Statement}

The authors have no conflicts of interest to declare.

\section{Funding Sources}

No funding was received. 


\section{Author Contributions}

Y.T. treated the patient, including surgery and follow-up, collected the data, analyzed the ophthalmological findings, gave critical suggestions, prepared the figures, and prepared the report.

T.S. treated the patient, including surgery, and prepared the report.

N.I., S.Y., and R.K. treated the patient.

S.S. revised and finalized the paper.

All authors agree to be accountable for all aspects of the work. All authors attest that they meet the current ICMJE criteria for authorship.

\section{References}

1 Ascaso FJ, Huerva V, Grzybowski A. Epidemiology, Etiology, and Prevention of Late IOL-Capsular Bag Complex Dislocation: review of the Literature. J Ophthalmol. 2015;2015:805706.

2 Gross JG, Kokame GT, Weinberg DV; Dislocated In-The-Bag Intraocular Lens Study Group. In-the-bag intraocular lens dislocation. Am J Ophthalmol. 2004 Apr;137(4):630-5.

3 Krèpštė L, Kuzmienė L, Miliauskas A, Janulevičienė I. Possible predisposing factors for late intraocular lens dislocation after routine cataract surgery. Medicina (Kaunas). 2013;49(5):229-34.

4 Raj P, Kumar K, Chandnani N, Agarwal A, Agarwal A. Secondary Angle-Closure Glaucoma Due to Posterior Synechiae of Iris Following Combined Phacoemulsification and 23-Gauge Transconjunctival Vitrectomy. Semin Ophthalmol. 2017;32(5):537-42.

5 Chang SH, Wu WC, Wu SC. Late-onset secondary pigmentary glaucoma following foldable intraocular lenses implantation in the ciliary sulcus: a long-term follow-up study. BMC Ophthalmol. 2013 Jun;13:22.

6 Mihora LD, Bowers PJ Jr, Blank NM. Acute angle-closure glaucoma caused by vitreous prolapse after neodymium:YAG posterior capsulotomy. J Cataract Refract Surg. 2004 Nov;30(11):2445-7.

7 Bochmann F, Stürmer J. Chronic and Intermittent Angle Closure Caused by In-The-Bag Capsular Tension Ring and Intraocular Lens Dislocation in Patients With Pseudoexfoliation Syndrome. J Glaucoma. 2017 Nov;26(11):1051-5.

8 Hyams SW, Friedman Z, Neumann E. Elevated intraocular pressure in the prone position. A new provocative test for angle-closure glaucoma. Am J Ophthalmol. 1968 Oct;66(4):661-72.

9 Ohta T, Toshida H, Murakami A. Simplified and safe method of sutureless intrascleral posterior chamber intraocular lens fixation: y-fixation technique. J Cataract Refract Surg. 2014 Jan;40(1):2-7.

10 Smiddy WE, Sawusch MR, O’Brien TP, Scott DR, Huang SS. Implantation of scleral-fixated posterior chamber intraocular lenses. J Cataract Refract Surg. 1990 Nov;16(6):691-6.

11 Oshika T, Nagahara K, Yaguchi S, Emi K, Takenaka H, Tsuboi S, et al. Three year prospective, randomized evaluation of intraocular lens implantation through 3.2 and $5.5 \mathrm{~mm}$ incisions. J Cataract Refract Surg. 1998 Apr;24(4):509-14.

12 Giansanti F, Tartaro R, Caporossi T, Murro V, Savastano A, Barca F, et al. Intraocular lens dislocation: a nobel in-situ scleral refixation technique using a 25 Gauge trocar in the anterior chamber. BMJ Open Ophthalmol. 2018 Nov;3(1): e000174. 


\section{Case Reports in Ophthalmology}

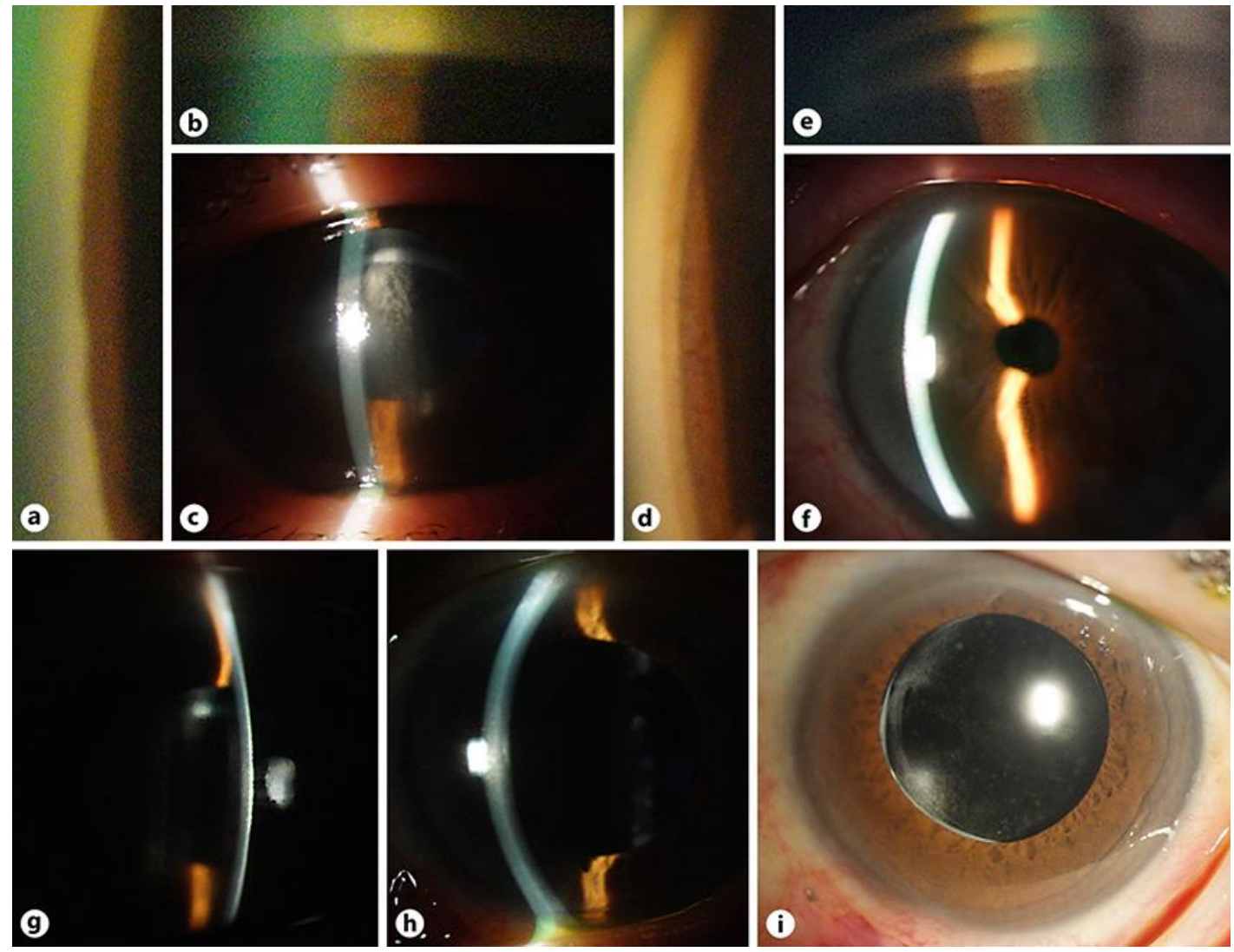

Fig. 1. Anterior eye feature. a, b Gonioscopy findings in the affected eye before mannitol drip infusion. c Anterior chamber and IOL in the affected eye before mannitol drip infusion. d, e Gonioscopy findings in the contralateral eye after mannitol drip infusion. $f$ Anterior chamber and IOL in the contralateral eye after mannitol drip infusion. g Slit-lamp photograph of the anterior eye segment at emergency consultation on December 18. $\mathbf{h}$ Slit-lamp photograph of the anterior eye at the last examination. $\mathbf{i}$ Diffused light photograph of the anterior eye at the last examination. 


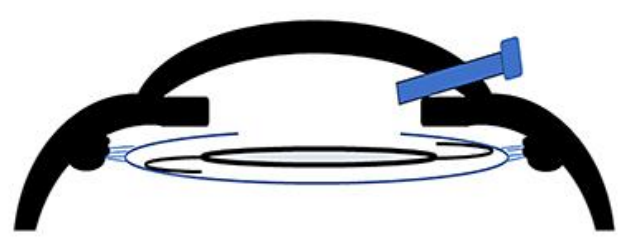

a

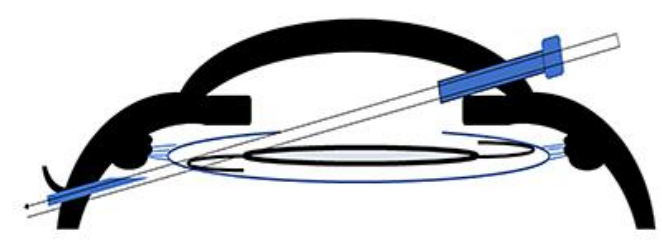

(c) 2020 The Author(s). Published by S. Karger AG, Basel www.karger.com/cop

Takada et al.: Repeating Increase in Intraocular Pressure by Instability of IOL

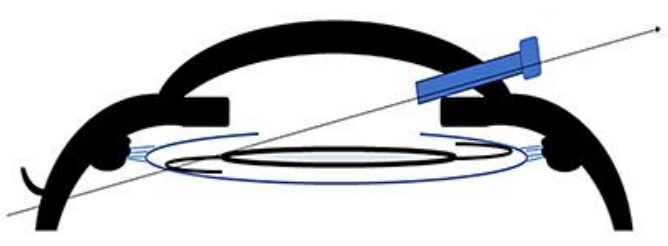

b

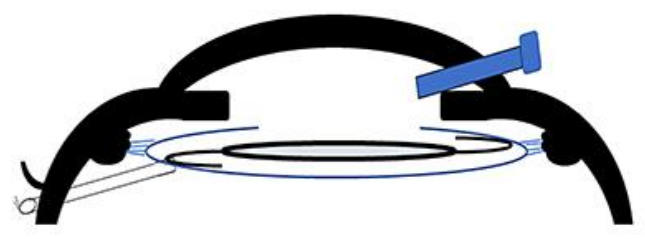

d

Fig. 2. Operation procedure. A 10-0 nylon thread that was connected to a straight needle at each end was used. a A $27 \mathrm{G}$ trocar was placed in the corneal limbus. b A 10-0 nylon thread-connected straight needle was advanced under the scleral flap, penetrated the IOL-capsule complex (between IOL optic and haptic), and was led out of the eye through a trocar. c Another side of the thread-connected straight needle was inserted again through the trocar, penetrated the IOL-capsule complex (outside the haptic part), and the straight needle was captured inside the $27 \mathrm{G}$ needle advanced under the scleral flap and guided out of the eye. $\mathbf{d}$ The 10-0 nylon thread was suture-fixed to the sclera under the scleral flap. 


\section{Case Reports in Ophthalmology}
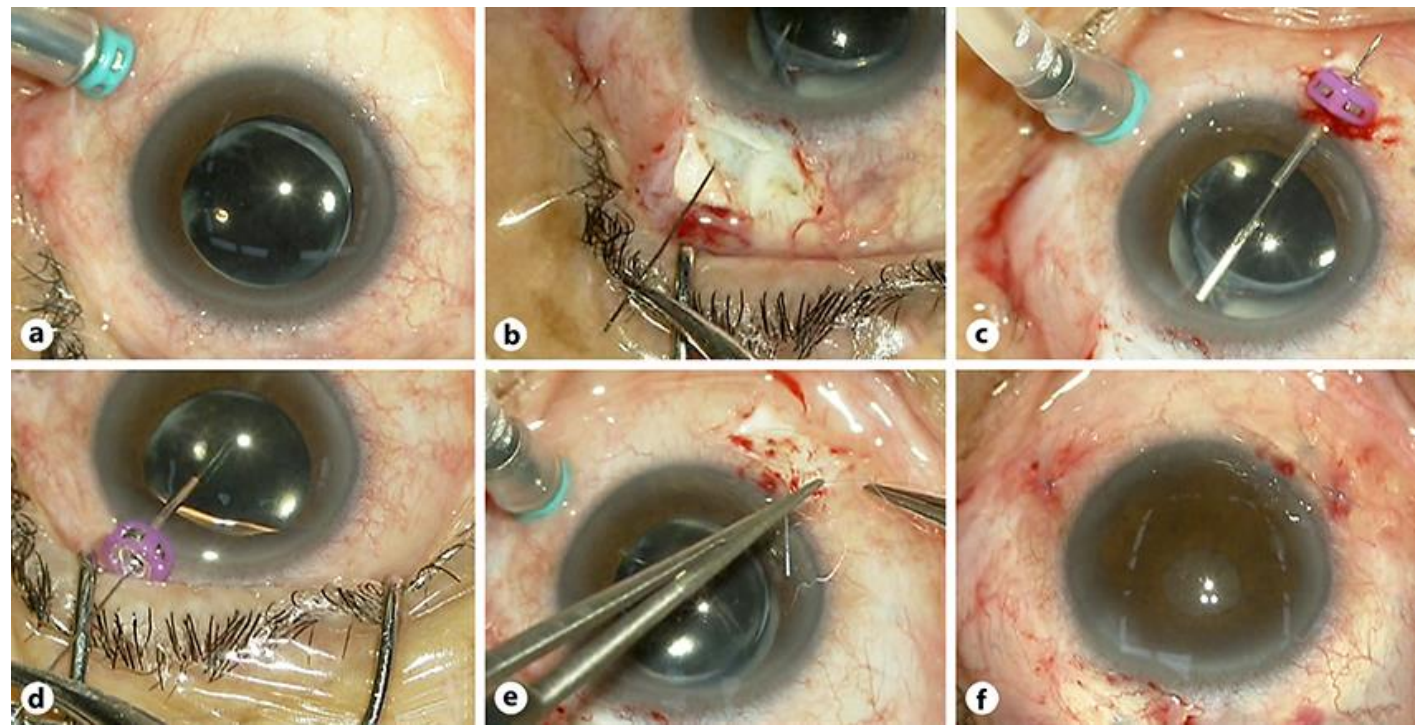

Fig. 3. Intraoperative photographs. a Placement of the irrigation port. $\mathbf{b}$ Puncture of the scleral tissue beneath the scleral flap with a 10-0 nylon thread-connected straight needle at the 1-o'clock direction. c After the straight needle was led out of the eye through a $27 \mathrm{G}$ trocar placed in the peripheral cornea, it was returned into the anterior chamber through the same $27 \mathrm{G}$ trocar, and a $27 \mathrm{G}$ needle was advanced under the scleral flap at the 1-o'clock direction. $\mathbf{d}$ On the opposite side, the IOL-lenticular capsule complex was also pierced with the straight needle and was threaded. e The 10-0 nylon thread was sutured under the scleral flap. $f$ The surgery is ended by removing the irrigation port and suturing the scleral flap and conjunctiva. 